\title{
Editorial: Special Issue on Organic Computing
}

Organic Computing has emerged recently as a challenging vision for future information processing systems, based on the insight that we will soon be surrounded by systems with massive numbers of processing elements, sensors and actuators. Because of the scale and complexity of these systems, it is infeasible to monitor and control them entirely from external observations; instead, they will need to widely monitor, control and adapt themselves. To do so, these components will need to be aware of their environment, to communicate freely, and to organize themselves in order to perform the required actions and services. The presence of such networks of intelligent systems in our environment opens up fascinating application areas but, at the same time, bears the problem of their controllability. Hence, these systems must be as robust, safe, and trustworthy as possible. In order to achieve all these goals, computing systems will have to act more independently, flexibly, and autonomously.

Currently, only living beings have these properties to a satisfactory degree. Therefore, Organic Computing puts special emphasis on systems that exhibit some life-like properties, such as being self-organizing, self-configuring, selfhealing, self-protecting, and context-aware. Many local processes of life are becoming better and better understood, but the organization of all these processes into a coherent and functional organic system requires further research both on experimental and theoretical aspects of organization. This is the view from which the name of Organic Computing originates. Its computing metaphors and guiding principles are directly informed by what is known about living systems.

Basic strategies and first applications of Organic Computing can be found in Würtz [2008]. The vision of Organic Computing and its fundamental concepts arose independently in different research areas like Neuroscience, Molecular Biology, and Computer Science and Engineering. Its engineering aspect overlaps considerably with Autonomic Computing [Murch 2006] and other selforganizing and self-adaptive computing paradigms (see, e.g., the special issue in volume 4 of this journal). Organic Computing is broader in scope than Autonomic Computing, which restricts its natural metaphor to the autonomic nervous system and its applications to computing services.

The dissociation from self-organizing and self-adaptive computing is naturally less sharp, as is their mutual distinction. In system construction, Organic Computing focuses on the integration of bottom-up emerging properties (selforganizing) and top-down design, which self-adapts its parameters. The final goal is the same-systems which reliably do not bother the developer with unnecessary details and yet produce an overall behavior within the desired confinements.

(C) 2010 ACM 1556-4665/2010/9-ART09 $\$ 10.00$

DOI 10.1145/1837909.1837910 http://doi.acm.org/10.1145/1837909.1837910

ACM Transactions on Autonomous and Adaptive Systems, Vol. 5, No. 3, Article 9, Pub. date: September 2010. 
For the special issue on Organic Computing, eight manuscripts were submitted. Three of them have been selected in a thorough two-tier review process and now form this issue.

The first article [Schmeck et al. 2010] directly approaches the conflicting objectives of creating self-organizing systems and being able to control the result. To that end, the authors study different methods of integrating multiple observer-controller architectures. They propose a metric to quantify the autonomy of given systems and measure their degree of self-organization.

Fekete et al. [2010] presents an application of Organic Computing to the control of highway traffic. The central structure is the hovering data cloud, an abstract data collection that is dynamically created in a traffic jam and supported by the ever-changing set of cars that make up the congestion. They show in simulations that decisions made by these clouds can significantly improve the throughput of traffic.

In the third article [Grushin and Reggia 2010], an artificial-intelligence system for the self-assembly of desired structures is improved by a mechanism that aims at generating a production systems with few rules, which in turn leads to short assembly times. This is an example of autonomously growing structures, which are nevertheless constrained by an overall plan.

All articles restrict their application scope to computer simulations of the targeted real systems. This indicates that the methods of Organic Computing are still in their infancy and have yet to prove viable in the real world. Practical application has partly been achieved in the time since the manuscripts were submitted. Also, some of the experiences gained in these studies are expected to generalize well to real applications.

We thank the editor-in-chief for the opportunity to edit this special issue and for her help and support along the way. We thank the authors for their contributions and their patience during the reviewing process. Thanks also go to the reviewers for working hard in order to guarantee the quality of this issue. Finally, we are grateful for funding from the German Research Foundation (DFG) in the priority program "Organic Computing."

\author{
ROLF P. WÜRTZ \\ Ruhr-University Bochum \\ Rolf.Wuertz@ini.rub.de \\ KiRstie L. Bellman \\ The Aerospace Corporation \\ Kirstie.L.Bellman@notes.aero.org \\ HARTMUT Schmeck \\ Karlsruhe Institute of Technology \\ Hartmut.Schmeck@kit.edu \\ ChRistian IGEL \\ Ruhr-University Bochum \\ Christian.Igel@ini.rub.de
}




\section{REFERENCES}

Fekete, S. P., Schmidt, C., Wegener, A., Hellbrück, H., And Fischer, S. 2010. Empowered by wireless communication: Distributed methods for self-organizing traffic collectives. ACM Trans. Autonom. Adapt. Syst. 5, 3.

Grushin, A. ANd RegGiA, J. A. 2010. Parsimonious rule generation for a nature-inspired approach to self-assembly. ACM Trans. Autonom. Adapt. Syst. 5, 3.

Murch, R. 2006. Autonomic Computing. IBM Press Prentice-Hall.

Schmeck, H., Müller-Schloer, C., ÇaKar, E., Mnif, M., and Richter, U. 2010. Adaptivity and self-organisation in organic computing systems. ACM Trans. Autonom. Adapt. Syst. $5,3$.

WürTZ, R. P., Ed. 2008. Organic Computing. Springer. 